\title{
Confined gold nanoparticles enhance the detection of small molecules in label-free impedance aptasensors $\uparrow$
}

\author{
Ana S. Peinetti, ${ }^{a}$ Helena Ceretti, ${ }^{b, c}$ Martín Mizrahi, ${ }^{d}$ Graciela A. González, ${ }^{a}$ \\ Silvana A. Ramírez, ${ }^{b, c}$ Felix G. Requejo, ${ }^{d}$ Javier M. Montserrat ${ }^{\star b, c}$ and \\ Fernando Battaglini*a
}

A controlled architecture of nanoelectrodes, of a similar size to small molecule-binding aptamers, is synthesized inside nanoporous alumina. Gold nanoparticles with a controlled size (about $2 \mathrm{~nm}$ ) are electrogenerated in the alumina cavities, showing a fast electron transfer process toward ferrocyanide. These uncapped nanoparticles are easily modified with a thiol-containing aptamer for label-free detection of adenosine monophosphate by electrochemical impedance spectroscopy. Our results show that the use of a limited electrical conducting surface inside an insulating environment can be very sensitive to conformational changes, introducing a new approach to the detection of small molecules, exemplified here by the direct and selective detection of adenosine monophosphate at the nanomolar scale.

Received 4th March 2015, Accepted 18th March 2015 DOI: $10.1039 / \mathrm{c} 5 \mathrm{nr} 01429 \mathrm{~h}$ www.rsc.org/nanoscale generated antibodies can be used as recognition elements. These equilibrium-based sensors are widely used; however, this approach presents two important requirements. Firstly, the antigen has to be recognized by the immune system, which is practically useless for small molecules. Secondly, the signal generation depends on the introduction of a label; a common practice is a sandwich assay where a second labeled antibody binds to the analyte. Therefore, the synthesis of a labeled antibody is needed for signal generation, and again the size of the analyte plays a relevant role in the binding of the second antibody.

Different strategies have been developed for the label-free detection of biomolecules, ${ }^{5}$ where electrochemical impedance spectroscopy (EIS) plays a relevant role. In EIS, the electron transfer rate of a probe is measured as a resistance $\left(R_{\mathrm{et}}\right)$ on a surface modified with a recognition element, for example an antibody. When the antibody-antigen complex is formed, the electron transfer rate of the probe changes with the antigen concentration, ${ }^{6}$ without the need for a second labeled antibody. This change is mainly due to the size of the analyte which prevents the probe from reaching the surface for the electron transfer process.

An alternative to the use of antibodies has been the development of aptamers by molecular evolution techniques. These molecules are usually defined as single-stranded oligonucleotides composed of RNA or DNA with molecular recognition properties, ${ }^{4 a}$ although the same term is used for peptide ligands obtained from phage display techniques. ${ }^{7}$ Keeping the focus on oligonucleotide sensors, they present several advantages as recognition agents: no animals are needed for their
${ }^{a}$ INQUIMAE (CONICET) - Departamento de Quimica Inorgánica, Analitica y Quimica 
synthesis; during the chemical synthesis, modifications can be introduced at defined positions; they can be developed for virtually any molecule, among them small molecules, where adenosine, its nucleotides and cocaine have been extensively used as model systems. ${ }^{4 b}$

Several ingenious works regarding the combination of aptamers with EIS for the label-free detection of proteins can be cited. ${ }^{8}$ However, as pointed out by Willner and Zayats, ${ }^{4 a}$ the use of EIS as a means to identify affinity complexes between aptamers and small molecules is a difficult issue. The formation of the aptamer-small molecule complex on the electrode yields a minute, usually undetectable, change in the interfacial electron transfer rate.

The general approach to overcome this limitation is the use of aptamer sequences longer than the recognition region or complementary strands, to induce a detectable structural change. ${ }^{4}$ Alternatively, other way to detect these small changes could be to reduce the surface of the electrode to a size close to the aptamer. In this way, even minimal changes in the closeness of the electrode surface will have an important effect in the interfacial electron transfer process.

In a recent publication, the construction of gold nanoelectrodes inside a porous alumina matrix has been presented. In this system, gold nanoparticles (AuNPs) are formed by electrochemical reduction inside the porous alumina where the interfacial electron transfer is limited to the gold surface. ${ }^{9}$ This nanoelectrode array shows excellent catalytic and electron transfer properties with a well defined structure (Scheme 1, left).

Adenosine monophosphate (AMP, MW: $347.2 \mathrm{Da}$ ) is directly linked to the energy metabolism of cells, contributing to the main pathway for adenosine formation, ${ }^{10}$ which in turn is involved in different neural processes in the brain like sleep, arousal and locomotion. ${ }^{11}$ Due to its clinical relevance and size, AMP constitutes a model system as a small molecule. Its aptamer was one of the first synthesized, ${ }^{12}$ and its conformational changes and recognition sites were widely studied. The AMP-binding aptamer is a sequence of 27 nucleotides that can be pictured as having two possible structural states: either a random coil or a stem-loop structure. The random-coil state can be represented as a flexible cord of $1 \mathrm{~nm}$ in diameter and $10 \mathrm{~nm}$ in length. When the aptamer recognizes AMP, two

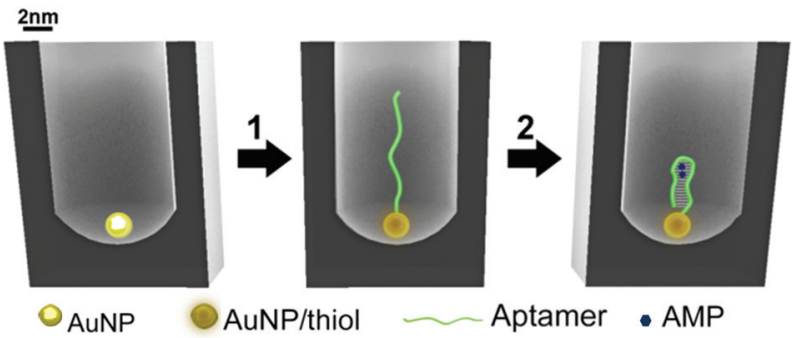

Scheme 1 Representation of an alumina pore. Step 1: Co-adsorption of the aptamer and hexanethiol. Step 2: AMP recognition. AuNP and pore diameter are in scale. molecules are intercalated at adjacent sites within a rectangular widened groove, changing the aptamer conformation to a stem-loop structure, with a $2 \mathrm{~nm}$ diameter ${ }^{13,14}$ In terms of a regular electrode, these are slight conformational changes to hinder the access of a redox probe to the surface.

In this work we decided to explore the potential of controlled sized $2 \mathrm{~nm}$ AuNPs confined in alumina to build an aptamer-based label-free sensor able to detect small molecules, taking AMP as a model system. As the AMP-binding aptamer has a similar size to that of the AuNP, the expected conformational changes will have an important impact on the signal generation. Scheme 1 outlines the concept in this work, The aptamer is co-adsorbed with hexanethiol (Scheme 1, center); then, the modified system exposed to AMP shows a conformational change affecting the coverage of the AuNP (Scheme 1, right) and therefore the probe access to the surface. This hypothesis is confirmed by EIS experiments showing a sensitive response at the nanomolar scale to AMP. The observed results are explained taking into account the dimensional changes produced by the aptamer and compared with results obtained using bulk gold electrodes modified in the same way, showing the advantages of controlling the system architecture at the nanometer scale.

\section{Experimental section}

\section{Materials}

All reagents were of analytical grade. Water $\left(18 \mathrm{M} \Omega \mathrm{cm}^{-1}\right)$ was provided by Millipore Simplicity equipment. Oligonucleotide sequences were desalted and HPLC purified (Sigma-Genosys). The AMP aptamer sequence was the original Szostack sequence $^{12}$ plus five additional thymidines as linkers. The scramble sequence was designed with the same amount of bases as the aptamer but in random positions.

Aptamer sequence:

5'-[ $\mathrm{C}_{6} \mathrm{H}_{13}$-S-S- $\left.\mathrm{C}_{6} \mathrm{H}_{12}\right]$ TTTTTACCTGGGGGAGTATTGCGGAGGAAGGT-3,

Scramble sequence:

$5^{\prime}$-[ $\mathrm{C}_{6} \mathrm{H}_{13}$-S-S- $\left.\mathrm{C}_{6} \mathrm{H}_{12}\right]$ ACGAAGGGCGGGGGTAGGGTATTTACG-3'. Screen printed bulk gold electrodes (SPGE) were prepared as previously reported. ${ }^{15}$

\section{Nanoporous alumina and gold nanoparticle synthesis}

Working electrodes $\left(4 \mathrm{~mm}^{2}\right)$ were prepared from aluminum 1145 (99.5\%). Surface pretreatment was carried out by degreasing the surface in an ultrasonic bath with acetone, followed by electropolishing in a $5: 1$ ethanol- $\mathrm{HClO}_{4}$ solution $(\mathrm{v}: \mathrm{v})$ at $18 \mathrm{~V}$ for 1 minute. The cleaned surface was immediately exposed to an acid electrolyte $\left(15 \% \mathrm{H}_{2} \mathrm{SO}_{4}\right)$ at room temperature $(15 \mathrm{~V}$, 1 minute), using a lead plate as a counter electrode in front of the working electrode. Once the electrode was anodized, it was left for 5 minutes in the acid electrolyte and then rinsed with MilliQ water. The pore size and depth were characterized by scanning electron microscopy. 
Gold electrodeposition was carried out using an electroplating commercial solution containing $15 \mathrm{~g} \mathrm{~L}^{-1} \mathrm{~K}\left[\mathrm{Au}(\mathrm{CN})_{2}\right]$ as the electrolyte. Anodized aluminum and a gold plate were used as the working and counter electrodes, respectively. Gold electrodeposition was performed in three steps: (i) metal deposition at $-3 \mathrm{~mA} \mathrm{~cm}{ }^{-2}$ for $8 \mathrm{~ms}$; (ii) application of $3 \mathrm{~mA} \mathrm{~cm}-2(2 \mathrm{~ms})$ to decrease the capacitive oxide layer and interrupt the electric field at the interface where it is being deposited; (iii) no current is applied for $500 \mathrm{~ms}$, to recover the ion concentration in the pores by diffusion from the solution. In total, 3000 cycles were performed.

FESEM micrographs. Micrographs were taken using a field emission scanning electron microscope (FESEM, Zeiss DSM 982 Gemini) at the Advanced Center for Microscopies (CMA, Universidad de Buenos Aires). Pore size distribution was calculated using ImageJ software.

$\mathrm{X}$-ray techniques. Nanoparticle size and sulfur coverage in the nanoparticles were determined by Extended X-Ray Absorption Fine Structure (EXAFS). Au $\mathrm{L}_{3}$-edge EXAFS spectra were measured at room temperature in fluorescence mode at the XAFS2 beam line at the Laboratorio Nacional de Luz Síncrotron (LNLS, Campinas, Brazil). An ionization chamber was used to detect the incident flux and a 15-element germanium solid state detector was used to sense the fluorescence signal from the sample. Data were processed using ATHENA with the AUTOBK background removal algorithm. ${ }^{16}$ The spectra were calibrated using a metallic film of gold. The EXAFS oscillations $\chi(k)$ were extracted from the experimental data with standard procedures using the ATHENA program. ${ }^{16}$ The $k^{2}$ weighted $\chi(k)$ data, to enhance the oscillations at higher $k$, were Fourier transformed. The Fourier transformation was calculated using the Hanning filtering function. EXAFS modeling was carried out using the ARTEMIS program which is part of the IFFEFIT package. ${ }^{16,17}$

Electrochemical measurements. Impedance measurements were carried out in a $\mu$ Autolab type III potentiostat provided with software for data acquisition. Typical electrode modification was carried out as follows: a $1 \mu \mathrm{M}$ disulfide aptamer solution in MilliQ water was reduced with $1.5 \mathrm{mM}$ tris(2-carboxyethyl)phosphine (TCEP) in Tris buffer, $\mathrm{pH}$ 7.4, for 2 hours at room temperature in the dark. The reduced oligonucleotide was incubated with the electrode for 30 minutes at room temperature. The electrode was rinsed with buffer and then immersed in a solution containing $50 \mathrm{mM} \mathrm{K}_{4}\left[\mathrm{Fe}(\mathrm{CN})_{6}\right]$ in $50 \mathrm{mM}$ HEPES buffer ( $\mathrm{pH} 7.4$ ) as the redox probe. EIS was performed at the formal potential of the redox probe, $0.20 \mathrm{~V} v s$. $\mathrm{Ag} / \mathrm{AgCl}$ using a frequency range from $10 \mathrm{kHz}$ to $1 \mathrm{~Hz}$, and the voltage amplitude was $10 \mathrm{mV}$. Then, the electrode was rinsed with buffer and exposed to a solution of a given AMP concentration in Tris buffer, $\mathrm{pH}$ 7.4, for 15 minutes at room temperature. Each AMP concentration was determined in triplicate and in each case a new electrode was used. A new EIS measurement was carried out by rinsing the electrode with buffer and immersing it in the ferrocyanide solution. Data were represented as Nyquist plots. An equivalent circuit was used to fit the impedance spectroscopy and to determine electrical para- meter values for each experiment. The equivalent circuit included the following elements: the electrolyte resistance $\left(R_{\mathrm{S}}\right)$ in series with two branches in parallel; one corresponding to the electron transfer resistance $\left(R_{\mathrm{et}}\right)$ and the other one to a constant phase element (CPE), representing the non-ideal behavior of the double layer as a capacitor. The difference between $R_{\mathrm{et}}$ after and prior to AMP incubation was used as signal.

Quartz balance measurements (QCM-D). The QCM-D experiments were performed using a Q-Sense instrument (QCM-D, Q-Sense E1, Sweden) equipped with Q-Sense Flow Module (QFM 401). For all measurements QSX 301 gold sensors were used. Samples were perfused using a peristaltic microflow system (ISMATEC, ISM 596D Glattbrugg, Switzerland). Gold sensors were cleaned with $\mathrm{O}_{3}$ and UV for 15 min immediately prior to use. The aptamer was reduced as before and the immobilization was performed as follows: $400 \mu \mathrm{L} 1 \mu \mathrm{M}$ reduced aptamer solution in MilliQ water, was injected and incubated for $30 \mathrm{~min}$ (with no flow) in order to allow $\mathrm{Au}-\mathrm{S}$ chemisorption. Unbound aptamer was removed by rinsing with buffer for $15 \mathrm{~min}$ at $100 \mu \mathrm{L} \mathrm{min}{ }^{-1}$. Injection of $1 \mu \mathrm{M}$ AMP was carried out, passing $400 \mu \mathrm{L}$ of the solution at a flow rate of $100 \mu \mathrm{L} \mathrm{min}{ }^{-1}$; then, the flow was interrupted for $15 \mathrm{~min}$ to allow the system to equilibrate. Buffer was injected for $15 \mathrm{~min}$ at $100 \mu \mathrm{L} \mathrm{min}{ }^{-1}$ to rinse unbound AMP.

Measurements were recorded at several odd multiples of the fundamental frequency (overtones) and frequency shifts were normalized by dividing by the overtone number. Data corresponding to the $3^{\text {rd }}$ overtone is shown in Fig. 3.

\section{Results and discussion}

Sulfuric acid was used as an electrolyte to obtain the nanoporous alumina structure. Fig. 1 shows the FESEM micrograph obtained; on the left, the top view of the oxide layer can be observed, from which the size and distance between nanopores can be estimated. The statistical analysis yields a $11 \pm 1 \mathrm{~nm}$ pore diameter and a $35 \pm 4 \mathrm{~nm}$ interpore distance. The cross-sectional view (right) shows the depth $(1 \mu \mathrm{m})$ and the regularity of the pores. ${ }^{9}$

The nanoelectrode array was built by exposing the nanoporous structure $\left(\mathrm{Al} / \mathrm{Al}_{2} \mathrm{O}_{3}\right)$ to a gold plating bath, and a pulsed electrodeposition sequence was applied to form gold nanoparticles $\left(\mathrm{Al} / \mathrm{Al}_{2} \mathrm{O}_{3} / \mathrm{AuNP}\right)$. As these AuNPs are a clean metallic surface, they can undergo an efficient electron transfer process and can be easily modified. Different strategies for sulfur anchoring to gold surfaces have been studied for the construction of aptamer based sensors. ${ }^{14,18,19}$ In this work, the immobilization strategy used was the co-adsorption of the aptamer with hexanethiol to prevent the overcrowded immobilization of the oligonucleotides.

The size and modification of the generated AuNPs were determined in situ by extended X-ray absorption fine structure (EXAFS) at the $\mathrm{AuL}_{3}$-edge. This X-ray absorption technique provides information on the local environment of $\mathrm{Au}$ atoms, 


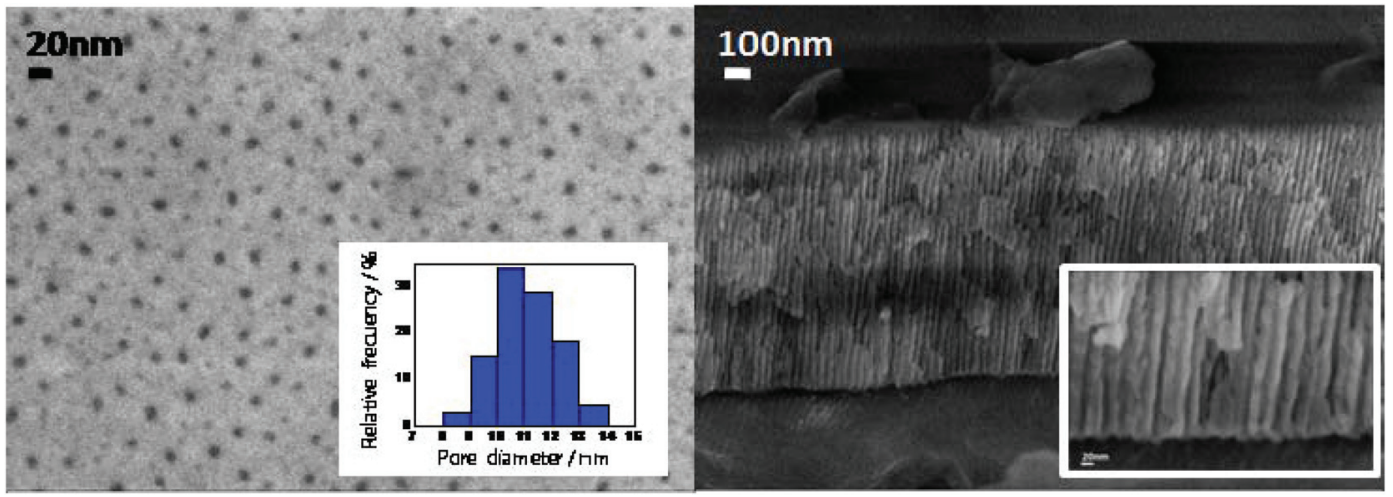

Fig. 1 FESEM images of the top (left) and cross-sectional view (right) of the nanoporous alumina grown on Al 1145 with $15 \%$ sulfuric acid as an electrolyte. The inset in the left figure shows the size distribution of the pores $(n=106)$; the inset on the right, a detailed image of the bottom of the mesoporous layer.

i.e. the number, type and distances between neighbouring $\mathrm{Au}$ without altering the geometry of the sample used for the electrochemical reaction. EXAFS results of samples $\mathrm{Al} / \mathrm{Al}_{2} \mathrm{O}_{3} / \mathrm{AuNP}$ and $\mathrm{Al} / \mathrm{Al}_{2} \mathrm{O}_{3} / \mathrm{AuNP} / \mathrm{SR}$ ( $\mathrm{R}$ representing either the aptamer or the hexane moieties) are shown in Fig. 2. For the sample $\mathrm{Al} / \mathrm{Al}_{2} \mathrm{O}_{3} / \mathrm{AuNP}$ only a coordination shell of $\mathrm{Au}$ around the absorber $(\mathrm{Au})$ atoms is present (thin line); while for the thiol modified sample a new peak attributed to the S-Au bond is observed (bold line). Nonlinear least squares fit of the theoretical EXAFS signal to the experimental data allows us to estimate different parameters regarding the structure of the AuNPs (details are given in ESI†). From this analysis, a $2.3 \mathrm{~nm}$ diameter for bare AuNPs was obtained from the fitted coordination number $(\mathrm{N})$ for the $\mathrm{Au}-\mathrm{Au}$ coordination shell, assuming spherical geometry for the NPs. ${ }^{20}$ Two coordination shells around the $\mathrm{Au}$ atoms are present in the sample $\mathrm{Al} / \mathrm{Al}_{2} \mathrm{O}_{3} / \mathrm{AuNP} /$ SR. One at $2.34 \AA$ corresponding to sulfur atoms $(\mathrm{Au}-\mathrm{S})$ and the other one at $2.83 \AA$ corresponding to the first gold coordi-

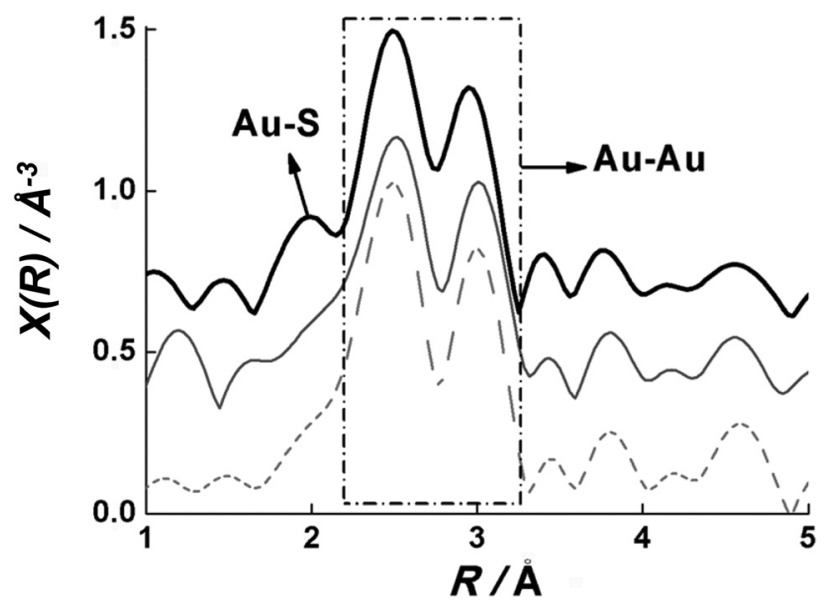

Fig. 2 Fourier transform of the EXAFS oscillation (without phase correction) for $\mathrm{Al} / \mathrm{Al}_{2} \mathrm{O}_{3} / \mathrm{AuNP} / \mathrm{SR}$ (bold line), $\mathrm{Al} / \mathrm{Al}_{2} \mathrm{O}_{3} / \mathrm{AuNP}$ (thin line) and bulk gold (dashed line). nation shell $(\mathrm{Au}-\mathrm{Au})$. Again, the $\mathrm{NP}$ average diameter was determined by the same procedure giving a similar result of about $2.3 \mathrm{~nm}$ diameter for the Au core of the capped NP. It is well established that the ratio of alkanethiol molecules to gold atoms in capped AuNPs is higher than that of the flat $\mathrm{Au}(111)$ crystal surface, i.e. $\mathrm{S}: \mathrm{Au}=1: 3$. Considering the experimentally obtained $\mathrm{S}: \mathrm{Au}$ ratio in hexanethiol-capped AuNPs with a similar diameter to $\mathrm{Al} / \mathrm{Al}_{2} \mathrm{O}_{3} / \mathrm{AuNP} / \mathrm{SR}$, the $\mathrm{S}: \mathrm{Au}$ ratio should be $0.65 .{ }^{21}$ Taking into account the fitted Au-S coordination number obtained here (see Table I, ESI $\dagger$ ) and the $\mathrm{S}$ : Au ratio, the AuNP sulfur coverage is $c a .60 \%$. This represents a high sulfur coverage, certainly higher than that obtained on the bulk $\mathrm{Au}(111)$ surface, which suggests that most of the surface exposed to the solution has been modified.

Fig. 3 shows the EIS behavior for $\mathrm{Al} / \mathrm{Al}_{2} \mathrm{O}_{3} /$ AuNP (squares) and after thiol modification (triangles), evidencing that the surface is partially blocked by the thiol compounds. Further

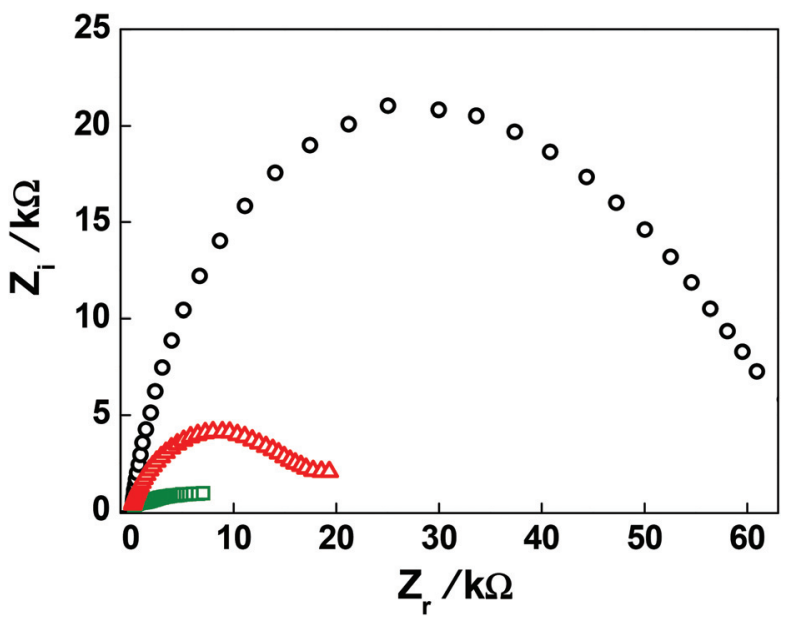

Fig. 3 Nyquist plots for the EIS experiments. Probe: $50 \mathrm{mM} \mathrm{Fe}(\mathrm{CN})_{6}{ }^{4-}$

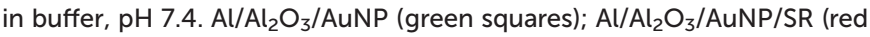
triangles); and $\mathrm{Al} / \mathrm{Al}_{2} \mathrm{O}_{3} / \mathrm{AuNP} / \mathrm{SR}-\mathrm{AMP}(500 \mathrm{nM}$ ) (black circles). 
exposure of $\mathrm{Al} / \mathrm{Al}_{2} \mathrm{O}_{3} / \mathrm{AuNP} / \mathrm{SR}$ to $500 \mathrm{nM}$ AMP produces a dramatic change in the impedance response (circles), which could be rationalized by the aptamer conformational changes. As it was previously explained, this aptamer can present two possible structural states: either a random coil or a stem-loop structure. The random-coil state can be represented as a flexible cord of $1 \mathrm{~nm}$ in diameter and $10 \mathrm{~nm}$ in length (Scheme 1, center). ${ }^{14}$ Considering the AuNP as a sphere of $2.3 \mathrm{~nm}$ in diameter, the maximum surface available will be $16 \mathrm{~nm}^{2}, c a .100$ $\mathrm{Au}$ atoms. Even though the curvature of the surface could help to introduce more $\mathrm{S}$ atoms to the surface, ${ }^{21}$ the size of the aptamer and the repulsion between the negatively charged phosphodiester groups suggests that only a few aptamer molecules are immobilized on each AuNP. When the aptamer recognizes AMP, two analyte molecules are intercalated at adjacent sites within a rectangular widened groove, changing the aptamer conformation to a stem-loop structure (Scheme 1, right). This new structure is more rigid and has a higher negative charge density near the surface. ${ }^{13,14}$ The mild change in the electron transfer resistance observed after the aptamerhexanethiol coadsorption suggests that very few aptamers are immobilized surrounded by the short alkyl thiol (yielded by the disulfide aptamer modification). When the AMP is recognized, the aptamer configuration change on this small confined surface dramatically modifies the ability of the probe to access the AuNP.

To show the relevance of working with these confined AuNPs, the same modification was carried out on bulk gold electrodes. Surface changes were followed by QCM-D and impedance experiments. QCM-D allows us to monitor the adsorption process, accompanied by a frequency change of $14.7 \mathrm{~Hz}$ (Fig. 4). If only hexanethiol (MW: $134 \mathrm{Da}$ ) was adsorbed, and considering that a gold surface completely covered by alkyl thiols contains $7.7 \times 10^{-10} \mathrm{~mol} \mathrm{~cm}^{-2}, 22$ the frequency change would be $4 \mathrm{~Hz}$, which is less than $30 \%$ of the change observed here. Therefore, most of the frequency

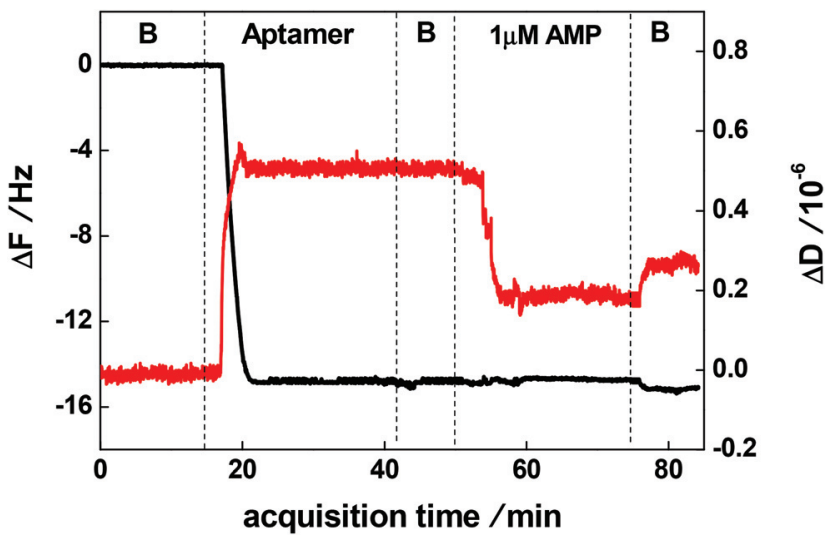

Fig. 4 QCM-D plot for the exposure of gold to the coadsorption of aptamer and hexanethiol followed by recognition of AMP. B represents the rinse steps with buffer. Frequency changes (black line, left axis); dissipation changes (red line, right axis).

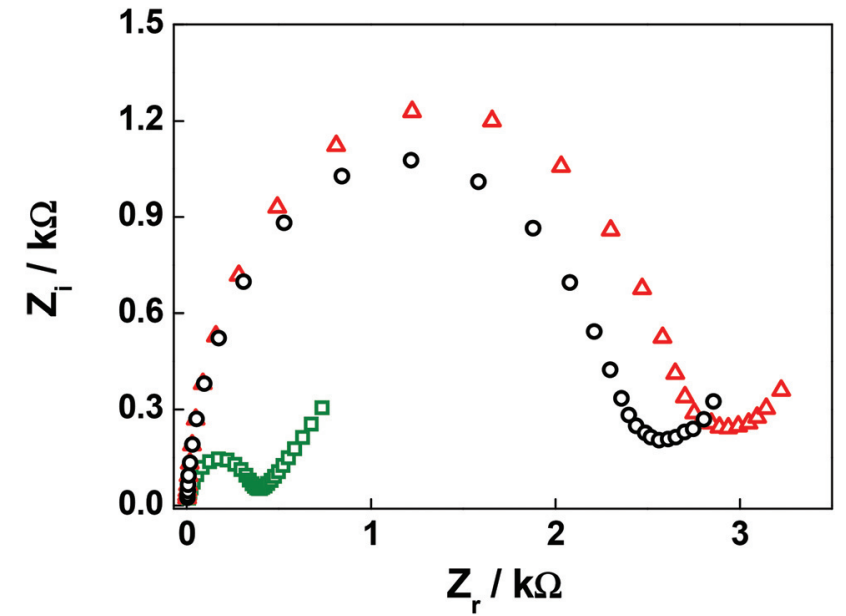

1

Fig. 5 Impedance response to $50 \mathrm{mM}$ ferrocyanide in buffer $\mathrm{pH} 7.4$, for $\mathrm{Au}$ (green squares); Au/SR (red triangles); Au/SR-AMP (black circles).

change can be attributed to the aptamer mass (MW: $10334 \mathrm{Da}$ ), representing a surface concentration of $2.4 \times 10^{-11}$

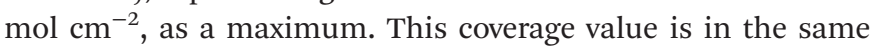
order as those previously reported, ${ }^{22,23}$ indicating that the aptamer reacts with less than $4 \%$ of the total gold atoms present in the bulk electrodes. On the other hand, dissipation measurements show a slight increase due to the presence of the free strands on the surface. ${ }^{24}$ Since AMP is a small molecule, its binding to the modified electrode cannot produce an important change in the frequency; however, the dissipation response decreased (Fig. 4, red trace). This change can be explained in terms of the aptamer contraction generating a rather compact structure on the surface.

EIS experiments for the modified bulk gold electrode showed a small change in the response when $500 \mathrm{nM}$ AMP was recognized (Fig. 5). The differences in the $R_{\mathrm{et}}$ can be explained due to the interplay between free space and charge repulsion, considering that the conformational state of the aptamer changes from a random coil to a stem-loop structure due to the AMP recognition. The conformational change folds the aptamers, making the strand more rigid. Therefore, the ability to repel the ferrocyanide probe by sweeping a given area of the electrode is partially lost, and the probe can get closer to the aptamer-free electrode surface decreasing the $R_{\mathrm{et}}$.

To establish the selectivity of the aptamer modified AuNPs to AMP, control experiments were carried out. Early studies
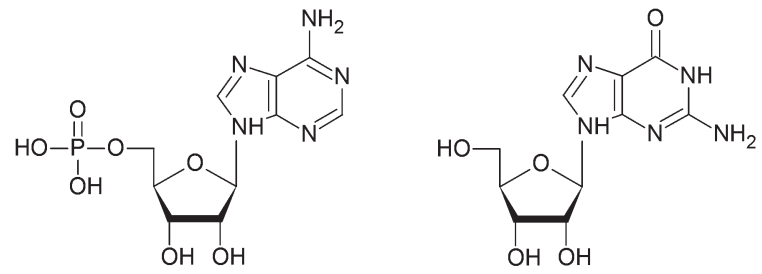

Fig. 6 Molecular structures of AMP (left) and guanosine (right). 
Table 1 Selectivity control experiments

\begin{tabular}{llrr}
\hline Sequence & Analyte & {$[$ Analyte]/nM } & $\Delta R_{\mathrm{et}} / \mathrm{k} \Omega$ \\
\hline Aptamer & AMP & 500 & $39 \pm 2$ \\
Aptamer & G & 1000 & $4 \pm 1$ \\
Scramble & AMP & 500 & $2 \pm 1$
\end{tabular}

regarding the recognition features of the DNA aptamer used in this work establish that adenosine, AMP and ATP are bound to the aptamer with practically the same binding constant. ${ }^{12,13}$ This is due to the fact that aptamer recognition involves interactions only with the adenosine moiety, i.e. with functional groups on the base and the sugar (Fig. 6, left) and not with the eventual phosphate moieties. Therefore, in our selectivity studies guanosine (Fig. 6, right), a nucleoside with a close structure, was used to study the selectivity of our assay.
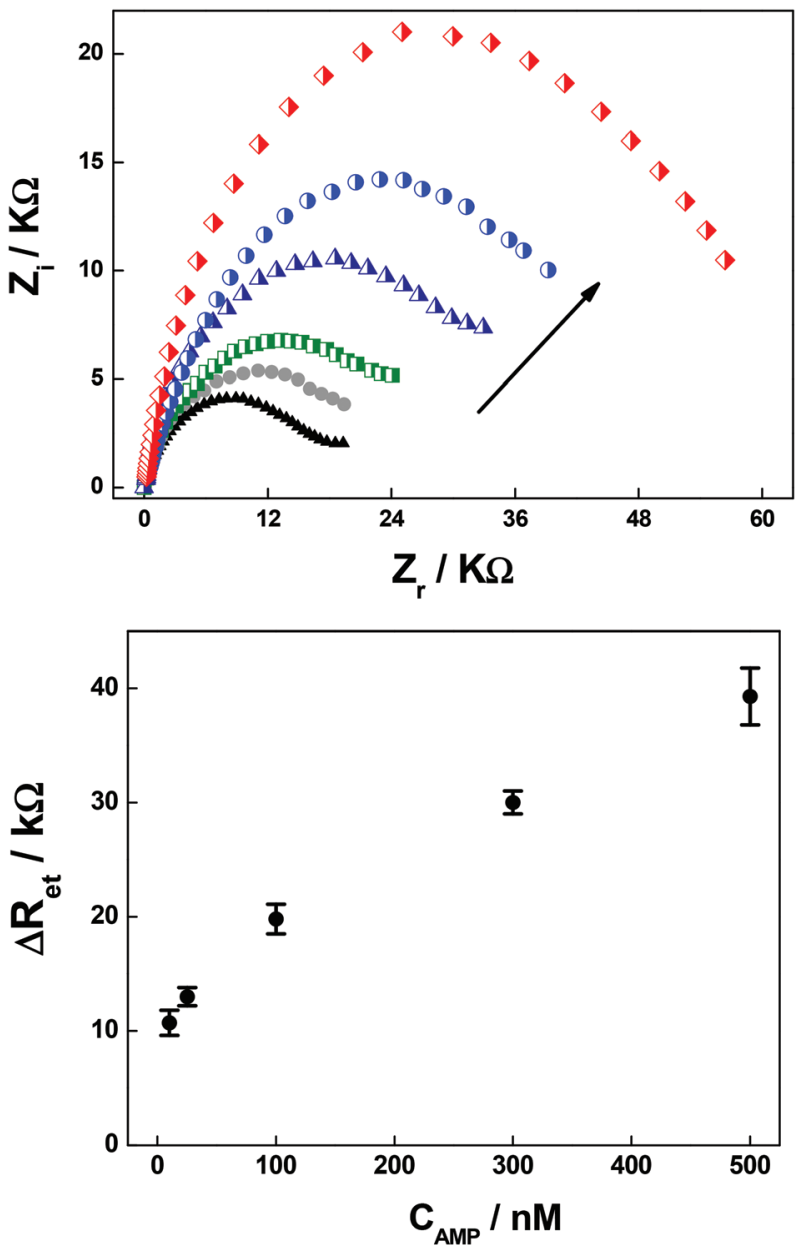

Fig. 7 Top: Nyquist plots for different AMP concentrations. The arrow indicates increasing concentrations $(0,10,25,100,300$ and $500 \mathrm{nM})$. Bottom: The effect of the AMP concentration on $R_{\text {et }}$ for the $\mathrm{Al} / \mathrm{Al}_{2} \mathrm{O}_{3} /$ AuNP/SR electrode. $\Delta R_{\text {et }}$ values are the measurements taken from three independent sensors for each concentration. $\Delta R_{\text {et }}=R_{\text {et }}\left(\mathrm{Al} / \mathrm{Al}_{2} \mathrm{O}_{3} / \mathrm{AuNP} /\right.$ $\mathrm{SR}-\mathrm{AMP})-R_{\mathrm{et}}\left(\mathrm{Al} / \mathrm{Al}_{2} \mathrm{O}_{3} / \mathrm{AuNP} / \mathrm{SR}\right)$. Errors bars indicate the standard deviation for three independent electrodes.
Table 1 shows the results for $\mathrm{Al} / \mathrm{Al}_{2} \mathrm{O}_{3} / \mathrm{AuNP}$ modified with the AMP-binding aptamer and guanosine (G) as the analyte. A negligible change establishes the selectivity of the system. Also, the $\mathrm{Al} / \mathrm{Al}_{2} \mathrm{O}_{3} / \mathrm{AuNP}$ modified with a random sequence oligonucleotide (scramble) was tested with AMP. Again, negligible changes were observed confirming that the recognition event and the conformational changes are responsible for the observed signal.

Finally, the sensitivity to AMP concentration was determined. EIS experiments were carried out at different AMP concentrations in a range from 10 to $500 \mathrm{nM}$ (Fig. 7, top). An increase in the impedance as the AMP concentration increases can be observed. Each AMP concentration was determined in triplicate, and in each case a new electrode was used. Using an equivalent circuit to fit the impedance spectroscopy (see Experimental section), the electron transfer resistance $\left(R_{\mathrm{et}}\right)$ was determined. The difference between $R_{\mathrm{et}}$ after and prior to AMP incubation was used as a signal (Fig. 7, bottom). Considering the dissociation constant of this aptamer, $6 \mu \mathrm{M},{ }^{25}$ the ability to detect this analyte at the nanomolar level can be considered as a remarkable sensitivity for this determination. ${ }^{26}$

\section{Conclusions}

The sensing of small molecules by direct molecular recognition represents a challenge for label free sensors, since the conformational changes undergone by the recognition element are limited to the adaptive folding around the analyte. In the case of a bulk gold electrode, this change affects only a fraction of the total area, a serious drawback in the sensor sensitivity. The approach taken in this work by the generation of isolated AuNPs in a porous alumina matrix overcomes this problem, since the dimensional changes produced by the recognition event are in the same scale as the surrounding environment, generating an important perturbation in the electron transfer process. This allows the sensitive detection of small molecules, represented here by AMP.

The use of the nanoengineered platform presented here can be extended to other analytes, since it does not require any special feature on the aptamer. Additionally, manipulation of the pore size could prevent electrode fouling by other species present in the sample.

Details regarding the fitting of X-ray experimental data are given in the ESI. $\dagger$

\section{Acknowledgements}

This work was financially supported by the following grants: PIP 01035 (CONICET, Argentina), XAFS1 beamline (LNLS, Brasil) proposal 17189, ANPCyT PICT-2011-0406, PICT-20110367, OPCW and UNGS. MM.,GAG., FR, JMM and FB are CONICET members. 
$1 \quad$ Notes and references

1 H. Shi, X. Ye, X. He, K. Wang, W. Cui, D. He, D. Li and X. Jia, Nanoscale, 2014, 6, 8754.

2 E. Busseron, Y. Ruff, E. Moulin and N. Giuseppone, Nanoscale, 2013, 5, 7098.

3 (a) S. Ziegler, V. Pries, C. Hedberg and H. Waldmann, Angew. Chem., Int. Ed., 2013, 52, 2744; (b) B. Mu, J. Zhang, T. P. McNicholas, N. F. Reuel, S. Kruss and M. S. Strano, Acc. Chem. Res., 2014, 47, 979.

4 (a) I. Willner and M. Zayats, Angew. Chem., Int. Ed., 2007, 46, 6408; (b) J. G. Walter, A. Heilkenbrinker, J. Austerjost, S. Timur, F. Stahl and T. Scheper, Z. Naturforsch., B: Chem. Sci., 2011, 67, 976.

5 H. K. Hunt and A. M. Armani, Nanoscale, 2010, 2, 1544.

6 E. Katz and I. Willner, Electroanalysis, 2003, 15, 913.

7 A. Gutés, B-Y Lee, C. Carraro, W. Mickelson, S-W Lee and R. Mabouduan, Nanoscale, 2013, 5, 6048.

8 (a) M. C. Rodriguez, A.-N. Kawde and J. Wang, Chem. Commun., 2005, 34, 4267; (b) A.-E. Radi, J. L. A. Sanchez, E. Baldrich and C. K. ÓSullivan, Anal. Chem., 2005, 77, 6320.

9 A. S. Peinetti, S. Herrera, G. A. González and F. Battaglini, Chem. Commun., 2013, 49, 11317.

10 T. Porkka-Heiskanen, L. Alanko, A. Kalinchuk and D. Stenberg, Sleep Med. Rev., 2002, 6, 321.

11 T. V. Dunwiddie and S. A. Masino, Annu. Rev. Neurosci., 2001, 24, 31.
12 D. Huizenga and J. W. Szostak, Biochemistry, 1995, 34, 1 656.

13 C. H. Lin and D. Patel, Chem. Biol., 1997, 4, 817.

14 Z. Li, L. Zhang, H. Mo, Y. Peng, H. Zhang, Z. Xu, C. Zheng and Z. Lu, Analyst, 2014, 139, 3137.

15 G. Priano, G. González, M. Günther and F. Battaglini, Electroanalysis, 2008, 20, 91.

16 B. Ravel and M. Newville, J. Synchrotron Radiat., 2005, 12, $537 \mathrm{e} 41$.

17 M. Newville, J. Synchrotron Radiat., 2001, 8, $322 \mathrm{e} 4$.

18 H. Ceretti, B. Ponce, S. Ramírez and J. M. Montserrat, Electroanalysis, 2010, 22, 147.

19 X. Zhang and V. K. Yadavalli, Biosens. Bioelectron., 2011, 26, 3142.

20 J. M. Ramallo-López, F. G. Requejo, A. F. Craievich, J. Wei, M. Avalos-Borja and E. Iglesia, J. Mol. Catal. A: Chem., 2005, 228, 299.

21 J. M. Ramallo-López, L. J. Giovanetti, F. G. Requejo, S. R. Isaacs, Y. S. Shon and M. Salmeron, Phys. Rev. B: Condens. Matter, 2006, 74, 073410, and references therein.

22 L. Strong and G. M. Whitesides, Langmuir, 1988, 4, 546.

23 (a) T. Ohshiro and M. Maeda, Chem. Commun., 2010, 46, 2581; (b) D. Y. Petrovykh, H. Kimura-Suda, L. J. Whitman and M. J. Tarlov, J. Am. Chem. Soc., 2003, 125, 5219.

24 G. Papadakis, A. Tsortos, F. Bender, E. E. Ferapontova and E. Gizeli, Anal. Chem., 2012, 84, 1854.

25 T. Hermann and D. J. Patel, Science, 2000, 287, 820.

26 J. Liu and Y. Lu, Angew. Chem., Int. Ed., 2006, 45, 90. 